\title{
CORRELACIÓN DEL SISTEMA DE CATALOGACIÓN DE LOS NÓDULOS TIROIDEOS TI-RADS Y LOS RESULTADOS DE LA BIOPSIA ASPIRADO CON AGUJA FINA EN PACIENTES ENTRE LAS EDADES DE 21 A 65 AÑOS EN UN HOSPITAL DE SANTO DOMINGO, REPÚBLICA DOMINICANA, DURANTE EL PERÍODO AGOSTO 2017- ENERO 2018
}

\begin{abstract}
Correlation between the classification system of thyroid nodules TI-RADS and the biopsy results by fine needle aspiration in patients between the ages of 21 to 65 years old in a hospital in Santo Domingo, Dominican Republic, during the period of August 2017- January 2018
\end{abstract}

\section{Jacquelín Díaza , Irvin Gonzalez ${ }^{\mathrm{b}}$, Lisely Tavarezc, Daniela Sosad ${ }^{\mathrm{d}}$ Alexis Cuevas ${ }^{\mathrm{e}}$, Daniela Calderón ${ }^{f}$, Alexa Estévez ${ }^{\mathrm{g}}$, Elena Kawas ${ }^{\mathrm{h}}$ y Carolina Ruiz}

Recibido: 3 de abril, $2020 \bullet$ Aprobado: 6 de julio, 2020

Cómo citar: Díaz J, Gonzalez I, Tavarez L, Sosa D, Cuevas A, Calderón D, Estévez A, Kawas E, Ruiz C. Correlación del sistema de catalogación de los nódulos tiroideos TI-RADS y los resultados de la biopsia aspirado con aguja fina en pacientes entre las edades de 21 a 65 años en un hospital de Santo Domingo, República Dominicana, durante el período agosto 2017- enero 2018. cysa [Internet]. 10 de junio de 2021 [citado 16 de junio de 2021];5(2):125-36. Disponible en: https://revistas.intec.edu.do/index.php/cisa/article/view/2218

\section{Resumen}

Introducción: uno de los motivos más frecuentes de consulta en el área de Endocrinología es la presencia de nódulos tiroideos. Esta patología, localizada en la glándula tiroides se estima que afecta del $4 \%$ al $7 \%$ de la población dominicana, teniendo predominio en mujeres.

Objetivo: correlacionar el sistema de catalogación de los nódulos tiroideos TI-RADS y los resultados de la biopsia por aspiración con aguja fina en pacientes de 21 a 65 años de edad en un hospital de Santo Domingo, República Dominicana, durante el período agosto 2017- enero 2018.

\footnotetext{
a Endocrinóloga. Instituto Tecnológico de Santo Domingo (INTEC). Santo Domingo, República Dominicana. ORCID: 0000-0001-9984-2395, Correo-e: diazjacqr31@icloud.com

b Estudiante de Medicina. (INTEC). ORCID: 0000-0001-8130-055, Correo-e: irvin21_07@hotmail.com

${ }^{c}$ Estudiante de Medicina. (INTEC). ORCID: 0000-0002-4229-2780, Correo-e: liselytavarez@gmail.com

${ }^{\mathrm{d}}$ Estudiante de Medicina. (INTEC). ORCID: 0000-0003-3631-4098, Correo-e: dany_sosa97@hotmail.com
}

\begin{abstract}
Introduction: One of the most frequent motives of consultation in the Endocrinology area is the presence of thyroid nodules. This pathology, located in the thyroid gland is estimated to affect $4-7 \%$ of the Dominican population, more predominant in women. OBJECTIVE: Correlate the classification system of thyroid nodules TI-RADS and the biopsy results by fine needle aspiration in patients between the ages of 21 to 65 years old in a hospital in Santo Domingo, Dominican Republic, during the period of august 2017January 2018.

\footnotetext{
${ }^{e}$ Estudiante de Medicina. (INTEC). ORCID: 0000-0003-0513-0405, Correo-e: acvas360@gmail.com

${ }^{\mathrm{f}}$ Estudiante de Medicina. (INTEC). ORCID: 0000-0003- 1688-3365, Correo-e: alexaestevezg@gmail.com,

${ }^{\text {h }}$ Estudiante de Medicina. (INTEC). ORCID: 0000-0002-5451-8193, Correo-e: elenamkn98@gmail.com

${ }^{i}$ Estudiante de Medicina. (INTEC). ORCID: 0000-0003-4757-2383, Correo-e: carolinaruizgonzalez02@gmail.com,
} Correo-e: danimichelle25@gmail.com,

g Estudiante de Medicina. (INTEC). ORCID: 0000-0002-3454-858X,
\end{abstract}


Metodología: estudio retrospectivo, descriptivo, observacional y de corte transversal en el que se analizaron las historias médicas de 34 pacientes del área de endocrinología en un hospital.

Resultados: el índice de correlación entre el sistema de catalogación de los nódulos tiroideos TI-RADS y los resultados de la biopsia por aspiración con aguja fina fue de $0 \%$, según el coeficiente de Kappa, mostrando un coeficiente de correlación bajo entre los dos estudios.

Conclusiones: la correlación entre los diagnósticos dados por los sistemas de catalogación difirió en su totalidad a la hipótesis nula, presentando un $0 \%$. El grupo etario que presentó mayor frecuencia de la patología tiroidea fue desde finales de la cuarta hasta la mitad de la quinta década de vida (48-56 años de edad), en un $57.14 \%$, con predominio de un $100 \%$ en el sexo femenino. La edad promedio de los pacientes que presentaron la patología tiroidea fue de 47 años.

Palabras clave: TI-RADS; BAAF; BETHESDA; nódulos tiroideos; biopsia histopatológica.

\section{Introducción}

Uno de los motivos más frecuentes de consulta en el área de Endocrinología es la presencia de nódulos tiroideos ${ }^{1}$. Esta patología, localizada en la glándula tiroides, es una neoplasia intratiroidea radiológicamente distinta al parénquima que la rodea ${ }^{2}$. Los nódulos tiroideos pueden ser coloides, adenomas, quistes, tiroiditis o carcinomas; hiperfuncionantes o hipofuncionantes; entre otros ${ }^{3}$. La principal causa de la formación de estos es aún desconocida, sin embargo, se conoce que son más frecuente en mujeres, en regiones con déficit de yodo, en personas mayores y en pacientes con antecedentes de irradiación en cabeza y cuello ${ }^{4}$. Se estima que afecta del $4 \%$ al $7 \%$ de la población dominicana, predominando en mujeres ${ }^{5}$. Algunos trastornos que también pueden causar la formación de nódulos son la falta de yodo, la presencia de adenomas que produzcan una insuficiencia o sobreproducción de hormonas tiroideas; presencia de calcificaciones o
Methodology: Retrospective, descriptive, observational, cross-sectional study about the analysis of the medical history of 34 patients of the Endocrinology area in a hospital.

Results: The correlation index between the classification system of thyroid nodules TI-RADS and the biopsy results by fine needle aspiration was $0 \%$, according to the Kappa coefficient, providing a low correlation coefficient between both studies.

Conclusions: The correlation between the diagnosis given by the classification systems differed in its entirety to the null hypothesis, presenting $0 \%$. The etary group that presented a higher frequency of thyroid pathology was from the end of the fourth decade to the fifth decade of life (48-56 years of age), with a $57.14 \%$, with the predominant of $100 \%$ in females. The mean age of patients with the thyroid pathology was 47 years old.

Keywords: TI-RADS; Fine Needle Aspiration Biopsy (FNA); BETHESDA; Thyroid Nodules; Histopathological Biopsy.

componentes sólidos en quistes tiroideos; la inflamación de la glándula tiroidea o tiroiditis; la formación de un bocio multinodular; o la existencia de un carcinoma en la glándula tiroides ${ }^{6}$.

Aunque esta patología es mayormente asintomática, en algunos casos, se presentan síntomas compresivos debido a la localización del nódulo7. Estos síntomas pueden deberse a la afectación de la tráquea (disnea, estridor, tos y sensación de ahogo); afectación del esófago (disfagia); en las venas yugular, subclavia o cava superior (disnea, estridor, distensión de las venas del cuello); compresión del nervio laríngeo recurrente (parálisis de cuerdas vocales); o compresión de la vía simpática cervical (síndrome de Horner) ${ }^{8}$. Otras manifestaciones serían quejas cosméticas (prominencia en la base del cuello observable a plena vista), dolor transitorio o crecimientos súbitos secundarios a hemorragia, o hipertiroidismo, esto último ocurriendo solo en el $1 \%$ de los casos $^{8}$. 
Esta neoplasia, es decir, un crecimiento anormal de tejido autónomo', requiere de distintos estudios para analizar su morfología y fisiología, que van desde la palpación tradicional del cuello, hasta el análisis de sangre de la Hormona Estimulante de Tiroides (TSH) y Tiroxina (T4), la observación de imágenes por la ecografía, captación de yodo según la gammagrafía y biopsia por aspiración con aguja fina ${ }^{10}$. La frecuencia de diagnóstico de un nódulo por palpación es del $4-7 \%{ }^{11}$. Su prevalencia en autopsias se encuentra entre el $8.2 \%$ y el $64.6 \%$, mientras que su detección ecográfica ha aumentado de un $19 \%$ a un $68 \%$ con el desarrollo tecnológico de los equipos de ultrasonido ${ }^{12}$.

Para observar la morfología del nódulo tiroideo, se debe realizar una ecografía tiroidea. Esta ecografía tiroidea determina el tratamiento y seguimiento que recibirán los nódulos. Sin embargo, esta puede mostrar distintos patrones ecográficos los cuales dificultan su catalogación. Debido a esto, Horvath et al. plantearon un sistema de clasificación denominado Thyroid Imaging Reporting and Data System (TI-RADS) para facilitar la catalogación de nódulos tiroideos ${ }^{12}$. Este cuenta con seis categorías que son: glándula tiroidea normal, benigno, probablemente benigno, sospechoso de malignidad, probablemente maligno y maligno ${ }^{12}$.

Una vez clasificada la ecografía en TI-RADS, da lugar a la biopsia por aspiración con aguja fina (BAAF), clasificado en el sistema de BETHESDA, siendo este proceso médico ideal debido a su precisión, al tener una sensibilidad del $83 \%$ y especificidad del $98 \%$, falsos negativos del $5 \%$ y falsos positivos del 1-7 \% 12. El sistema BETHESDA cuenta, al igual que el TI-RADS, con seis categorías que son: diagnóstico indeterminado, benigno, atipia de lesión folicular, neoplasia folicular, sospechoso de malignidad y maligno ${ }^{13}$.

A pesar de que ambos sistemas de catalogación cuentan con categorías similares, los resultados de la ecografía tiroidea, TI-RADS, no siempre concuerdan con los resultados de la biopsia por aspiración con aguja fina. A causa de esta incongruencia en los estudios, muchos pacientes terminan con un diagnóstico erróneo, lo que desencadena un problema, tanto para el hospital como para el paciente. Por esta razón, el estudio tuvo como objetivo determinar la correlación entre el sistema de catalogación de nódulos tiroideos TI-RADS y los resultados de la biopsia por aspiración con aguja fina en pacientes de 21 a 65 años de edad en un hospital de Santo Domingo, República Dominicana, durante el período de agosto 2017 - enero 2018, ya que debido a la incidencia de los nódulos tiroideos, es de suma importancia determinar qué tanto los resultados de ambos difieren, y así evitar diagnósticos errados.

\section{Materiales y métodos}

Este estudio fue de tipo retrospectivo, debido a que los datos fueron recolectados de fuentes secundarias durante el período de agosto 2017- enero 2018; asimismo, descriptivo y observacional, ya que los datos fueron detallados tal cual se presentaron sin ninguna intervención por parte de los investigadores; y de corte transversal, porque las variables fueron estudiadas en una sola ocasión, sin ofrecer continuidad una vez finalizada la recolección de datos.

La población estuvo compuesta por todos los pacientes diagnosticados con nódulos tiroideos que se realizaron la biopsia por aspiración con aguja fina y fueron clasificados con el sistema de catalogación de nódulos tiroideos TI-RADS, que asistieron a una consulta de Endocrinología en un hospital de Santo Domingo, República Dominicana de 21 a 65 años de edad durante el período de agosto 2017- enero 2018.

La herramienta de recolección de datos fue un formulario (véase anexo 1) creado por los autores y colaboradores, con el cual se obtuvo la información requerida de los récords médicos, facilitados por el departamento de archivos médicos, acerca de 
los pacientes que asistieron a una consulta de Endocrinología y presentaron nódulos tiroideos en un hospital de Santo Domingo. Con este se evaluó la relación entre los resultados del sistema de catalogación de nódulos tiroideos TI-RADS y los resultados de la biopsia por aspiración con aguja fina tomando como parámetro la biopsia histopatológica. En el formulario también fueron presentadas las variables de la investigación, que son sexo, grupo etario, tipo del nódulo tiroideo y tamaño del nódulo tiroideo. $\mathrm{El}$ acceso a estos datos fue acordado mediante un acuerdo de confidencialidad, en el cual se convino mantener el anonimato de los datos observados.

La relación entre los resultados de los sistemas de catalogación TI-RADS y BETHESDA fue comprobada al demostrar el nivel en el que los diagnósticos que proveen coinciden entre sí.

La muestra inicial del estudio fue de 34 pacientes, tomados al azar, teniendo en cuenta que fueran personas entre 21 y 65 ańos de edad, diagnosticados con nódulos tiroideos, catalogados por BETHESDA y por el sistema de categorización de nódulos tiroideos TIRADS. Luego de la recolección de datos, el estudio fue llevado a cabo con solo 7 pacientes, a causa de que 22 récords médicos contaban con los resultados de la sonografía, pero no fueron clasificados en TI-RADS, y los 5 restantes no fueron encontrados en el departamento de registro.

Para estimar las diferencias de los resultados según el sistema TI-RADS y el de BETHESDA se utilizó el índice de Kappa como cálculo estadístico para la comparación de tablas de contingencia ${ }^{14}$.

Los datos obtenidos fueron tabulados mediante Microsoft Excel versión Office 2016.

\section{Resultados}

Tabla 1. Relación entre el sistema de catalogación de nódulos tiroideos TIRADS y los resultados de la BAAF, en comparación con el diagnóstico histopatológico definitivo. $(\mathrm{N}=7)$

\begin{tabular}{|c|c|c|c|c|}
\hline \multicolumn{2}{|c|}{$\begin{array}{c}\text { Resultados de tres Sistemas } \\
\text { de Catalogación de Nódulos } \\
\text { Tiroideos }\end{array}$} & Positivo & $\begin{array}{c}\text { Tega- } \\
\text { tivo }\end{array}$ & \begin{tabular}{c} 
Hiopsia \\
tológica \\
\cline { 3 - 4 }
\end{tabular} \\
\hline \multirow{2}{|c|}{} & Positivo & $0(0 \%)$ & $0(0 \%)$ & Positivo \\
\cline { 2 - 4 } & $\begin{array}{c}\text { Nega- } \\
\text { tivo }\end{array}$ & $\begin{array}{c}7 \\
(100 \%)\end{array}$ & $0(0 \%)$ & Negativo \\
\hline
\end{tabular}

Fuente: Calderón, D., Estévez, A., Kawas, E., Ruiz, C., Jornada 78, BioINTEC, 2018.

La tabla anterior demuestra la frecuencia de diagnósticos positivos y negativos de malignidad para cada uno de los métodos de catalogación de nódulos tiroideos, y su relación con el diagnóstico definitivo provisto por la biopsia histopatológica. El $100 \%$ de la muestra fue catalogado como positivo por TI-RADS y negativo por BETHESDA, mientras que la biopsia histopatológica, de igual manera, cataloga a la muestra total como negativa de malignidad.

Para obtener estas informaciones, fue necesaria una sonografía para la categorización con el sistema de TI-RADS y la biopsia por aspiración con aguja fina para poder clasificar según el método de BETHESDA. La biopsia histopatológica fue realizada luego de una tiroidectomía o lobectomía, tanto parcial o total, para ambas. 
Figura 1. Presencia de nódulos tiroideos según sexo. $(\mathrm{N}=7)$

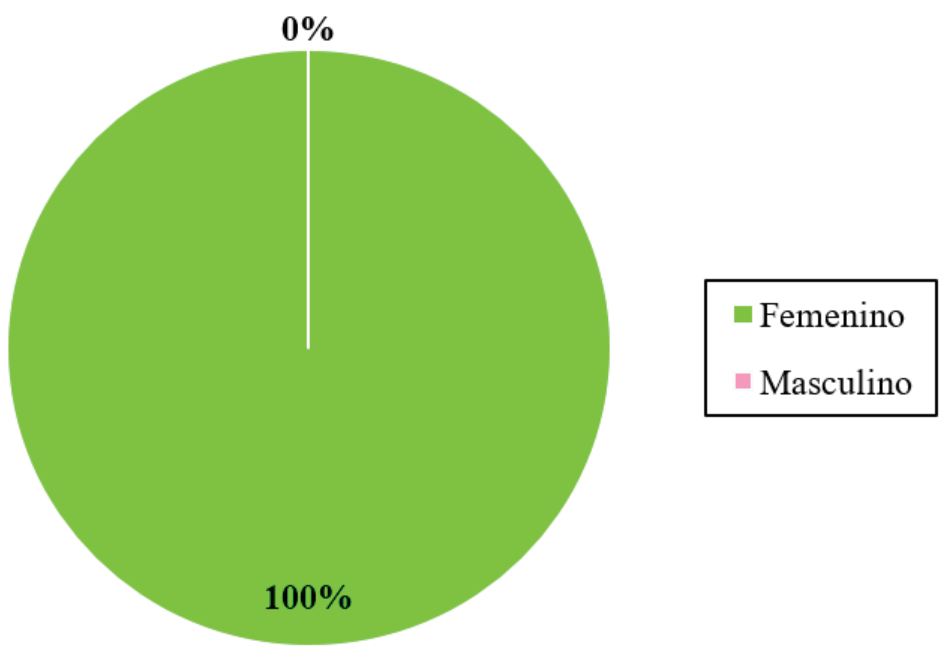

Fuente: Calderón, D., Estévez, A., Kawas, E., Ruiz, C., Jornada 78, BioINTEC, 2018.

De acuerdo con los resultados obtenidos, la presencia de patología tiroidea según el sexo, sobre un total de 7 casos, pertenece en su totalidad al sexo femenino
$(100 \%)$. En relación al sexo masculino, se observa un total de 0 casos $(0 \%)$.

Figura 2. Grupo etario. $(\mathrm{N}=7)$

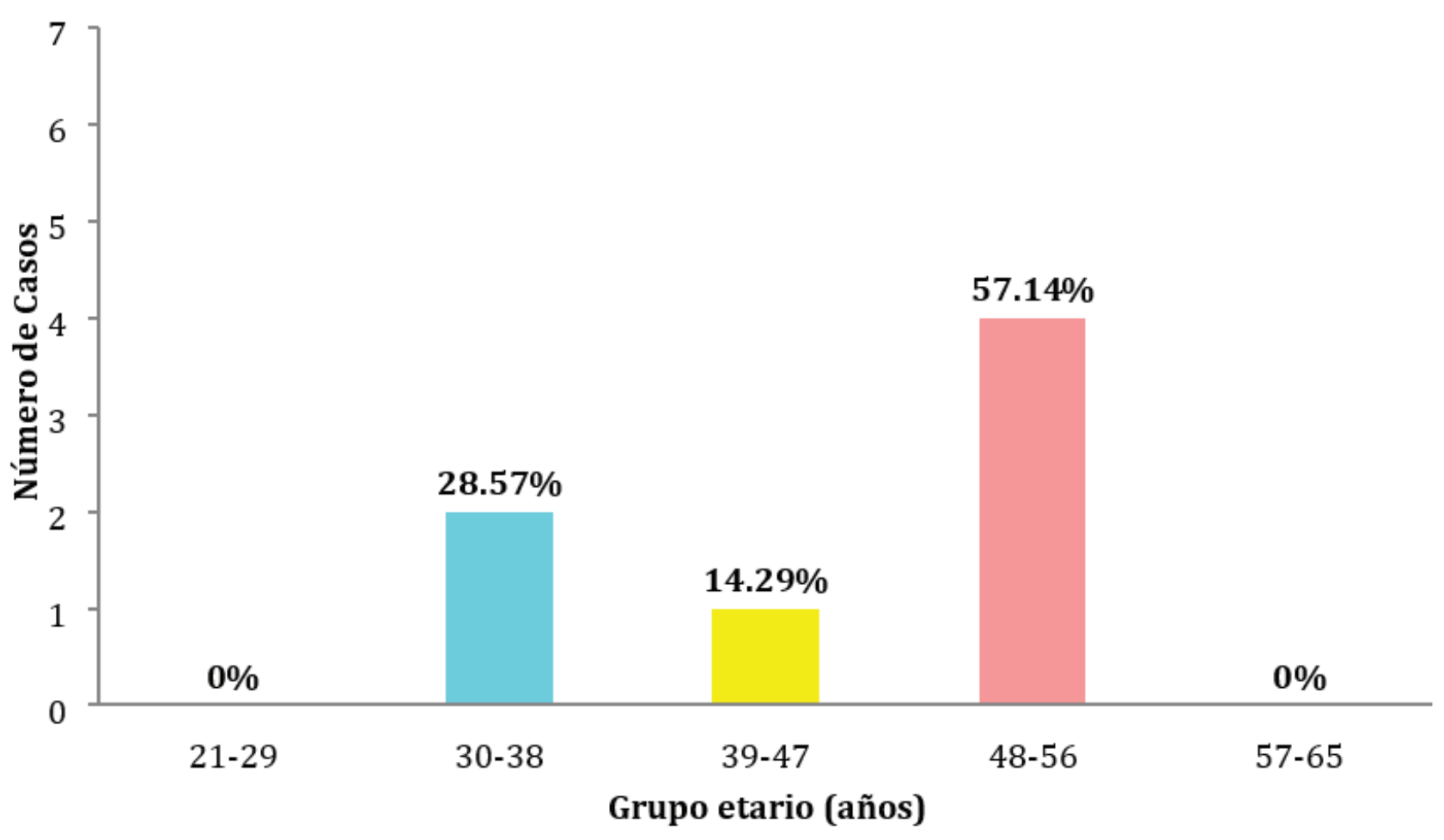

Fuente: Calderón, D., Estévez, A., Kawas, E., Ruiz, C., Jornada 78, BioINTEC, 2018. 
De acuerdo con los resultados obtenidos, se observa que la mayor cantidad de pacientes estudiados con presencia de nódulos tiroideos tienen una edad perteneciente al rango de $48-56$ años con un 57.14 $\%$ del total, correspondiendo a 4 casos. Mientras que hubo un $28.57 \%$ de los pacientes pertene- cientes al rango de 30-38 ańos, correspondiendo a 2 casos, y un $14.29 \%$ de pacientes pertenecientes al rango de 39 - 47 años, correspondiendo a 1 caso. Los pacientes restantes se reparten de igual manera en un $0 \%$ en el rango de $21-29$ ańos y de $57-65$ años, correspondiendo a ningún caso.

Figura 3. Distribución de nódulos tiroideos de acuerdo a su tipo histológico. $(\mathrm{N}=7)$

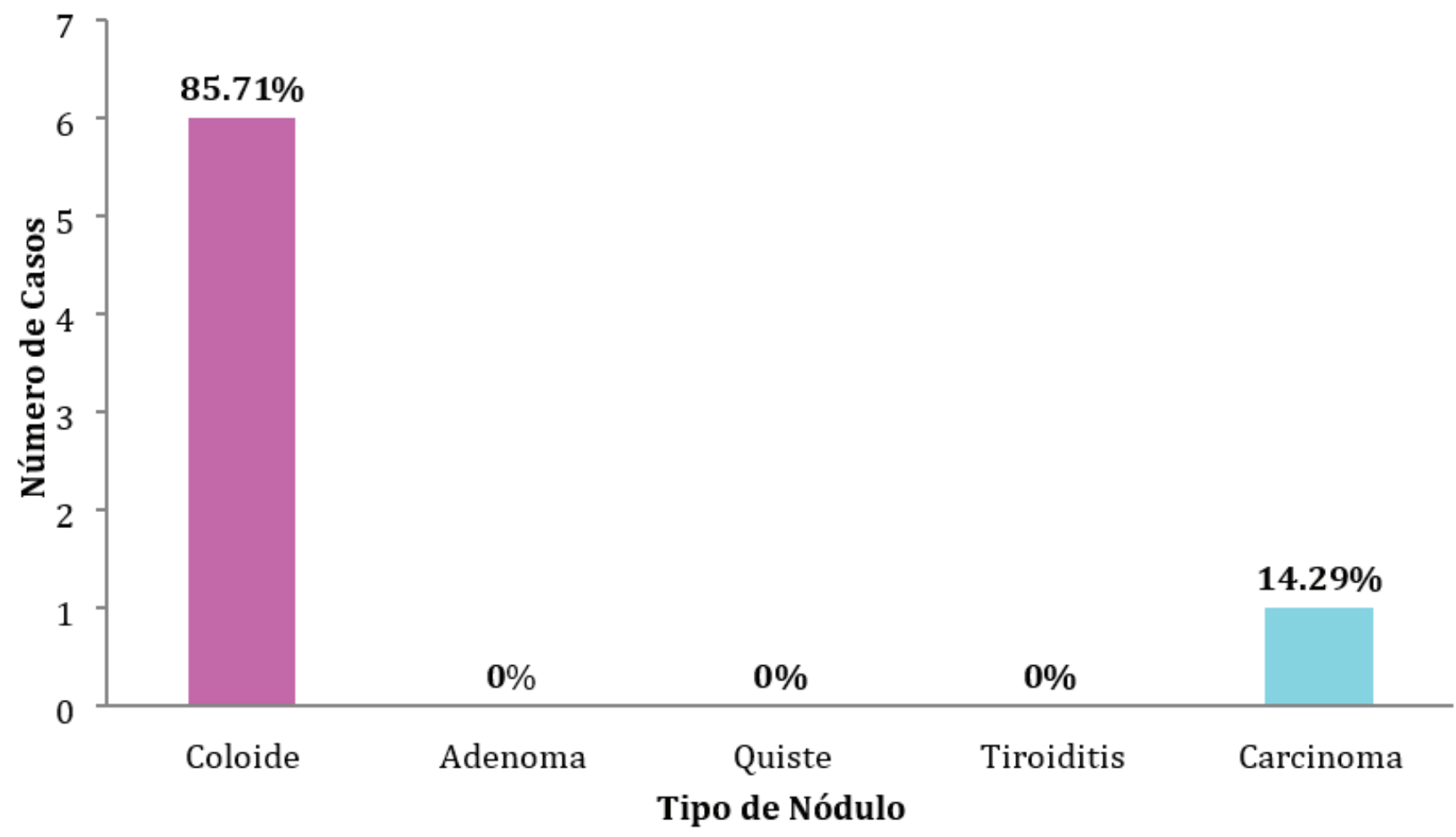

Fuente: Calderón, D., Estévez, A., Kawas, E., Ruiz, C., Jornada 78, BioINTEC, 2018.

Conforme a los resultados obtenidos a través del Los nódulos de tipo adenoma, quiste y tiroiditis diagnóstico definitivo, los nódulos coloides predominan, con un total de $85.71 \%$, equivalente a 6 casos. Los carcinomas presentaron el segundo mayor presentaron el mismo porcentaje correspondiente al $0 \%$, debido a que no fueron observados en ninguno de los casos. 
Figura 4. Distribución de nódulos tiroideos de acuerdo con su tamaño. (N=7)

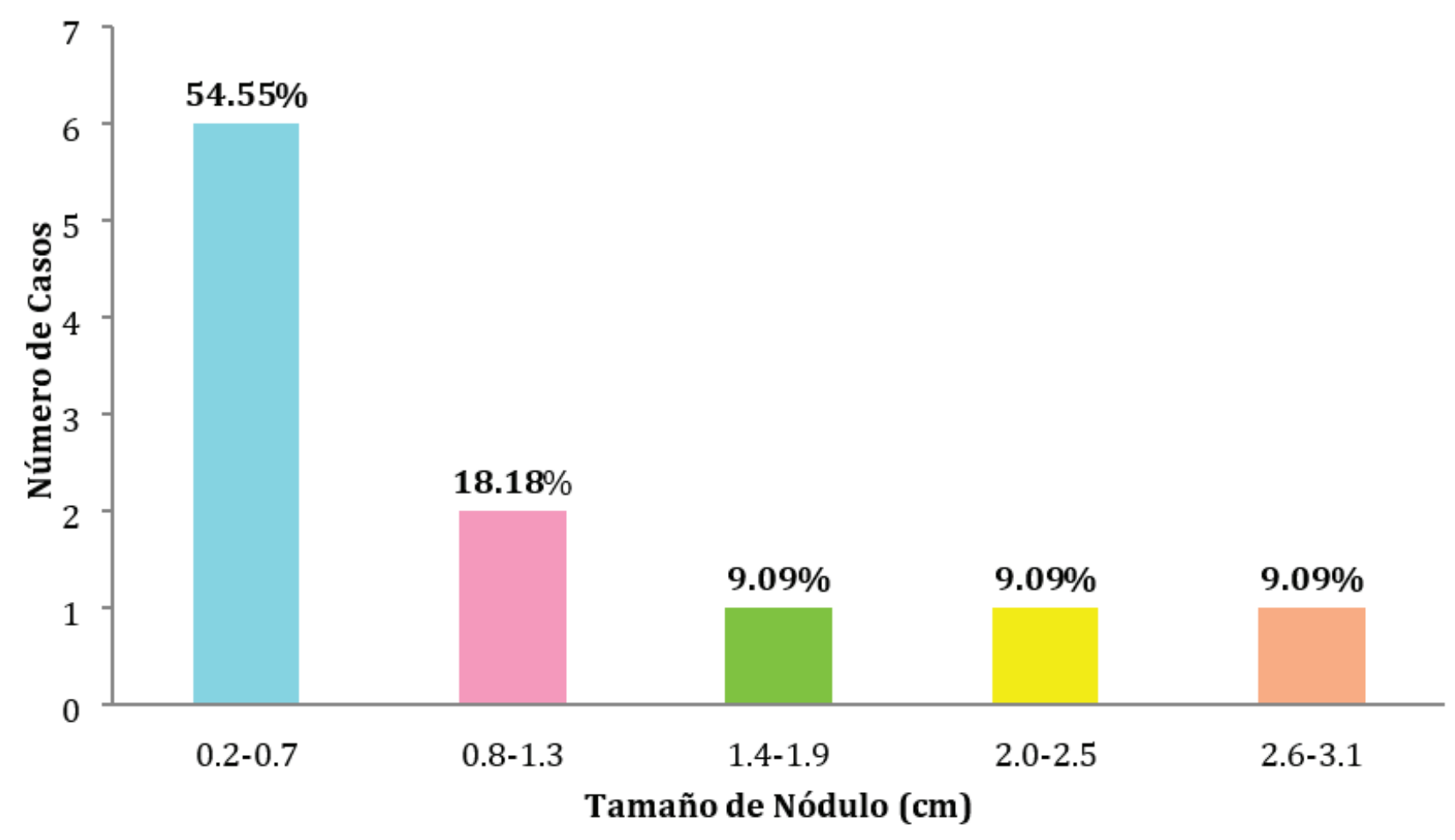

Fuente: Calderón, D., Estévez, A., Kawas, E., v, C., Jornada 78, BioINTEC, 2018.

Según los resultados obtenidos en el diagnóstico patológico definitivo, los nódulos tiroideos tienen un rango de tamaño que oscila entre $0.2-3.1 \mathrm{~cm}$. El tamaño dominante estaba entre $0.2-0.7 \mathrm{~cm}$, equivalente a $54.55 \%$ de la muestra observada. En segundo lugar, con respecto a su frecuencia, están los nódulos con un tamaño entre $0.8-1.3 \mathrm{~cm}$, equivalente a $18.18 \%$ de la muestra. Las dimensiones entre $1.4-1.9 \mathrm{~cm}, 2.0-2.5 \mathrm{~cm}$ y $2.6-3.1 \mathrm{~cm}$, presentan la misma frecuencia, equivalente a $9.09 \%$ de la totalidad de los casos.

\section{Discusión y conclusiones}

El sistema de catalogación de nódulos tiroideos TI-RADS y los resultados de la biopsia por aspiración con aguja fina presentaron correlación nula (0\%) (tabla 1). Según un estudio publicado por la Universidad del Rosario, en julio de 2015, intitulado Caracterización de Nódulos Tiroideos: Concordancia entre Estudio Citopatológico y Ecografía en la Detección de Nódulos Malignos's, del Dr. Héctor Blanco Ruiz, la concordancia entre los distintos sistemas de catalogación fue baja moderada, con un índice de Kappa de 0.55 de correlación, de un total de 100 pacientes investigados, lo cual difiere con los resultados obtenidos en la presente investigación.

La divergencia entre ambos resultados puede deberse a que ambas fueron realizadas en regiones geográficas distintas, donde los protocolos en cada estudio se siguen con diferentes grados de exigencia. A pesar de las diferencias, ambas investigaciones mostraron un nivel de correlación bajo. 
Una similitud entre la presente investigación y la mencionada anteriormente es la predominancia de nódulos tiroideos en mujeres (figura 1). Sin embargo, el tamaño de la población en el presente estudio influyó mucho en los resultados, debido a que solo contaba con 34 pacientes, de los cuales solo 7 aplicaban a los criterios de inclusión, y dentro de estos no se encontraban hombres.

Otro aspecto en el que las investigaciones presentaron incongruencias fue en el rango de edad de la población estudiada. El grupo etario que presentó mayor frecuencia de la patología tiroidea fue de 48-56 años de edad, en un 57.14\% (figura 2), mientras que en los resultados del estudio del Dr. Blanco Ruiz, la mayor frecuencia estuvo entre las edades de 13-16 ańos, en un 56.8 \%.

Ambas investigaciones discordaron en la predominancia de los tipos de nódulos, en el estudio realizado en la Universidad del Rosario, los nódulos malignos (carcinomas) tuvieron un $48 \%$ de frecuencia mientras que en la presente investigación solo tuvieron un 14.29 \% (figura 3). Esto puede deberse a la discrepancia en el rango de edades que tuvieron ambas investigaciones.

Otra de las diferencias que presentaron las investigaciones fue el tamaño predominante del nódulo tiroideo. Estaba entre $0.2-0.7 \mathrm{~cm}$, equivalente a $54.55 \%$ de la muestra observada en la presente investigación (gráfica 4) mientras que en la investigación previa el tamaño estaba entre $1.1-3.9 \mathrm{~cm}$.

Debido a que los sistemas de catalogación TI-RADS y BETHESDA son comúnmente utilizados en el tratamiento de nódulos tiroideos, se han hecho numerosas investigaciones para determinar la eficacia de ambas. En la presente investigación el índice de correlación entre el sistema de catalogación TI-RADS y los resultados de la biopsia por aspiración con aguja fina fue nulo (0\%), ya que ninguno de los casos estudiados concordó entre sí (tabla 1), indicando que ambos métodos no concuerdan.

Por lo que se llegó a la conclusión de que:

1. Es muy probable que exista una deficiencia en el sistema de clasificación de nódulos tiroideos TI-RADS, ya que BETHESDA es considerado el estándar de oro internacionalmente y sus resultados concordaron con los de la Biopsia Histopatológica, que es el análisis definitivo.

2. Existe una alta probabilidad de que el personal que realiza los estudios ecográficos no está debidamente equipado con las herramientas necesarias para catalogar el TI-RADS.

3. La discordancia se debe a la falta de rigidez por parte del hospital de exigir al personal seguir los protocolos de estudios sonográficos al clasificarlos según el TIRADS.

En caso de que la deficiencia se encuentre en el sistema de catalogación TIRADS, se debería considerar si este método de catalogación es una herramienta útil y eficaz en la práctica médica. Si la equivocación es de parte del personal que realiza los estudios ecográficos, se sugiere una capacitación estándar en el reporte sonográfico, para evitar discrepancias a la hora de catalogar y, así, lograr tener resultados más objetivos. Si se trata de una falla del hospital, se recomienda concientizar al personal que realiza los estudios ecográficos sobre la importancia de reportar en el informe radiológico la presencia o ausencia de cada una de las características ecográficas que se han relacionado con la probabilidad de malignidad en un nódulo tiroideo. Esto quiere decir, mencionar explícitamente qué categoría del TI-RADS presenta el nódulo tiroideo para así lograr una correcta catalogación del mismo y poder definir si es necesario o no la realización de una biopsia. De esta manera se evita la realización innecesaria de un procedimiento invasivo en un paciente. 


\section{Bibliografía}

1. Domínguez J, Baudrand R, Arteaga E, Campusano C, González G, Mosso L et al. Diseño de una escala ecográfica predictora de malignidad en nódulos tiroideos: Comunicación preliminar. Rev. méd. Chile [Internet].2009; 137(8):1031-6. Recuperado de https://scielo.conicyt.cl/scielo.php?script=sci_ arttext\&pid=S0034-98872009000800005\&ln$\mathrm{g}=\mathrm{es}$ [Citado 24 mayo 2018]

2. Román A, Restrepo L, Alzate C, Vélez A, Gutiérrez J. Nódulo tiroideo, enfoque y manejo. Revisión de la literatura [Internet]. Revista Iatreia. 2013;26(2):197-206. Recuperado de http:// www.scielo.org.co/pdf/iat/v26n2/v26n2a08. pdf [Citado 6 abril 2018]

3. Rivera R, Hernández S, Ochoa A, Rodríguez S, Torres P. Diagnóstico y tratamiento del nódulo tiroideo. Posición de la Sociedad Mexicana de Nutrición y Endocrinología,

4. A.C. Revista de Endocrinología y Nutrición [Internet]. 2010;18(1):34-50. Recuperado de http://www.medigraphic.com/pdfs/endoc/ er-010/er101g.pdf [Citado 6 abril 2018].

5. Mas A. Nódulo de tiroides, un problema muy frecuente. MasEndocrino. [Internet]. Recueprado de https://masendocrino.com/nodulo-de-tiroides/\#Cuales_son_las_causas_y_factores_de riesgo [Citado 6 abril 2018]

6. Instituto Tecnológico de Santo Domingo. Notas de Prensa. Enfermedad nodular de tiroides afecta mayormente a mujeres. [Internet]. Recuperado de https://www. intec.edu.do/prensa/ notas-de-prensa/item/enfermedad-nodular-detiroides-afecta-mayormente-a-mujeres [Citado 13 mayo 2018]

7. American Thyroid Association. Nódulos Tiroideos [Internet]. Recuperado de https:// www.thyroid.org/nodulos-tiroideos [Citado 6 abril 2018]

8. Verdecia Cañizares C, Portugués Días A, Longchong Ramos M. Alteraciones estructurales de la glándula tiroidea tras la radioterapia. Revistas Médicas Cubanas. [Internet].2009;81(1). Recuperado de http://scielo.sld.cu/scielo.php?script=sci_ arttext\&pid=S003475312009000100006\&ln$\mathrm{g}=\mathrm{es}$ [Citado 6 abril 2018]

9. Segura K, Buitrago M. Oncología: Nódulo Tiroideo. RevistaMédicadeCosta RicayCentroamérica [Internet]. 2016;73(620):465-8. Recuperado de http://www.binasss. sa.cr/revistas/ rmcc/620/art07.pdf [Citado 9 abril 2018]

10. Bogner P. Pathology and The Pathology of Neoplasia. Roswell Park Cancer Institute. [Internet]. Recuperado de https://www.roswellpark.org/sites/default/files/bogner_3_11_14. pdf [Citado 8 mayo 2018]

11. Lacic M. Nodulos tiroideos. Thyroid.Org. [Internet]. Recuperado de https://www.thyroid. org/wp-content/uploads/patients/brochures/ espanol/nodulos_tiroideos.pdf [Citado 12 mayo 2018].

12. Román-González A, Restrepo Giraldo L, Alzate Monsalve C, Vélez A, Gutiérrez Restrepo J. Nódulo tiroideo, enfoque y manejo. Revisión de la literatura. Scielo. [Internet] 2013;26(2). Recuperado de http://www.scielo.org.co/pdf/iat/ v26n2/ v26n2a08.pdf [Citado 15 mayo 2018].

13. Fernández Sánchez J. Clasificación TI-RADS de los nódulos tiroideos en base a una escala de puntuación modificada con respecto a los criterios ecográficos de malignidad. Revista Argentina de Radiología [Internet]. 2014;78(3):138-48. Recuperado de http://www.redalyc.org/articulo. oa?id=382533983004 [Citado 15 mayo 2018]. 
14. Pinto-Blázquez J, Del Valle-Manteca A, Solera-Arroyo J, Cuesta-Martínez L, Ursúa-Sarmiento I, Baizán-García M. Sistema Bethesda en el diagnóstico citopatológico de la patología de tiroides. Dialnet [Internet]. 2014;5(8):66-74. Recuperado de https://dialnet.unirioja.es/descarga/articulo/4756769.pdf [Citado 24 mayo 2018]

15. Vargas-Uricoechea H, Meza-Cabrera I, Herrera-Chaparro, J. Concordance between the TIRADSultrasoundcriteriaand theBETHESDA cytology criteria on the nontoxic thyroid nodule. Thyroid Research. [Internet]. 2017;10(1). Recuperado de https://www.ncbi.nlm.nih.gov/pmc/ articles/PMC5289008/\#!po=42.9688 [Citado 15 mayo 2018].
16. Blanco Ruiz, A. (2015). Caracterización de nódulos tiroideos: concordancia entre estudio citopatológico y ecografía en la detección de nódulos malignos (Tesis de maestría, Universidad El Rosario). Recuperado de https://repository. urosario. edu.co/bitstream/handle/10336/10627/ BlancoRuiz-Hector-2015.pdf? sequence $=1$ [Citado 6 abril 2018] 


\section{Anexos}

Anexo 1. Formulario de registro de información creado por los colaboradores

Correlación del sistema de catalogación de los nódulos tiroideos TI-RADS y los resultados de la biopsia por aspiración con aguja fina en pacientes de 21 a 65 años de edad en un hospital de Santo Domingo, República Dominicana durante el período agosto 2017- enero 2018

Sexo: Edad:

Fecha de consulta:

Resultados BAAF:

Resultados TI-RADS:

Resultados Biopsia Histopatológica:

Tipo de nódulo:

Tamaño de nódulo: 
Anexo 2. Acuerdo de confidencialidad

Correlación del sistema de catalogación de los nódulos tiroideos TI-RADS y los resultados de la biopsia por aspiración con aguja fina en pacientes de 21 a 65 años de edad en un hospital de Santo Domingo, República Dominicana, durante el período agosto 2017- enero 2018.

Fecha:

\section{ACUERDO DE CONFIDENCIALIDAD}

El Instituto Tecnológico de Santo Domingo (INTEC) está realizando una investigación estudiantil, BioINTEC. Esta tiene como objetivo verificar la concordancia que tiene el sistema de catalogación de nódulos tiroideos TI-RADS con el sistema de Bethesda tomando como parámetro la Biopsia Histopatológica. Este estudio proporcionará qué tanto los resultados de estas confirmaciones diagnósticas pueden diferir la una de la otra, para brindar un tratamiento eficaz y tomar las medidas de lugar. El estudio requiere información dentro de las historias médicas, por lo tanto, se requiere de la aprobación del centro hospitalario. De aceptar la participación en el estudio, se visualizarán las historias médicas de los pacientes para extraer los diagnósticos de la ecografía, la biopsia por aspiración con aguja fina y la biopsia histopatológica.

Entre los beneficios de esta investigación está la obtención de la concordancia entre el TIRADS y el sistema de BETHESDA, indicando al médico cuál método es certero y eficaz realizar como tratamiento para que se puedan eliminar y/o tratar el nódulo tiroideo por completo. Además, este estudio reduce la realización de procedimientos invasivos innecesarios en pacientes con poca probabilidad de presentar cáncer de tiroides mientras facilita la identificación de pacientes con mayor riesgo.

Toda la información proporcionada será confidencial y solo será manejada por parte de los colaboradores de la investigación. Si se llegaran a publicar los resultados de la investigación, tanto la identidad del paciente como el nombre de la institución se mantendrían en el anonimato. Se puede negar la participación en el estudio o retirarse cuando se considere necesario.

Se dio el tiempo de realizar cualquier pregunta sobre la investigación y todas fueron respondidas. De tener alguna pregunta o problema sé que me puedo comunicar con el INTEC. Al firmar este documento doy el consentimiento de que la institución participe en esta investigación.

Firma hospital:

Firma entrevistador: 